\title{
A Case of Eosinophilic Granulomatosis with Polyangiitis (Churg-Strauss, EGPA) Presenting With Relapsed Mononeuritis Multiplex
}

\author{
Hilmi BA a Ainon $M M^{b}$ \\ aDepartment of Internal Medicine, International Islamic University Malaysia (IIUM), Kuantan, Pahang, Malaysia \\ bDepartment of Internal Medicine, Hospital Tengku Ampuan Afzan, Kuantan, Pahang, Malaysia
}

\begin{abstract}
We report a case of eosinophilic granulomatosis with polyangiitis (EGPA), a rare multisystem disorder characterized by difficult-to-control asthma, hypereosinophilia and polyneuropathy. We also discuss the Five Factor Score (FFS) risk stratification strategy, which is used to quantitate the extent of the disease and guide treatment strategy.
\end{abstract}

KEYWORDS: Churg-Strauss syndrome, vasculitis, mononeuritis multiplex, cyclophosphamide

\section{INTRODUCTION}

Eosinophilic Granulomatosis with Polyangiitis (EGPA), formerly Churg-Strauss syndrome is a rare autoimmune disease characterized by the presence of asthma, small to medium vessel necrotizing vasculitis and eosinophilia. Diagnosis is mainly clinical with findings of asthma, chronic rhinosinusitis, eosinophilia and signs of vasculitis. Its clinical course usually evolves through three phases: the prodromal or allergic phase: hallmarked by asthma, sinusitis, or allergic rhinitis; the second or eosinophilic phase: characterized by peripheral eosinophilia as well as organ eosinophilic infiltration, for example eosinophilic gastroenteritis, and the third or vasculitic phase with clinical manifestations due to small-vessel vasculitis causing for example, peripheral neuropathy, cardiac failure, and cutaneous disease. However, not all patients develop all three phases or progress from one phase to the next in an orderly fashion. EGPA can involve any organ; however, the most common organ involved is the lung, followed by the skin. The typical case that should raise suspicion of EGPA is that of a patient with adult-onset asthma and a history of rhino-sinusitis,

Corresponding author:

Dr. Hilmi Bin Abdullah

MBBS, MMED (Internal Medicine)

Assistant Professor, Physician

Department of Internal Medicine

Kulliyyah of Medicine

International Islamic University Malaysia (IIUM)

Jalan Hospital

25100 Kuantan

Pahang

MALAYSIA

Email:drh_1@hotmail.com

Tel No: 609-5132797 who develops pronounced eosinophilia and lung infiltrates. Current treatment strategies have greatly reduced the morbidity and mortality associated with this rare disease.

\section{CASE REPORT}

A 45-year-old self-employed man with underlying bronchial asthma for 15 years was admitted to the medical ward with acute exacerbation of bronchial asthma. He had been receiving treatments from a district hospital for many years. His asthma was poorly controlled with recurrent hospital admissions of about once every 2-3 months within the past 2 years. However, there was no history of severe attack requiring intensive care admission or mechanical ventilation. He used to smoke for 8 years but quit soon after the diagnosis of asthma was made. He had no family history of asthma, nor any history of allergic rhinitis or eczema.

During the current admission, he also complained of numbness in both feet and left hand for the first time. Physical examination revealed wasting of the medial side of left palm and of the inner muscle of the left forearm. Left hand grip was weak. There was reduced sensation over the ulnar side of the left hand. Lower limb examination showed inability to dorsiflex the left foot (foot drop) and 'stocking pattern' sensory loss in both feet. Cranial nerve and right upper limb examination were normal. Other than evidence of bronchospasm, examination of other organ systems was normal.

His blood investigation results showed marked leukocytosis, predominantly eosinophilia (15.6 x $\left.10^{\circ} / \mathrm{l}\right)$, elevated erythrocyte sedimentation rate $(46 \mathrm{~mm} / \mathrm{hr})$ and mild transaminitis. Renal profiles 
were normal and his viral hepatitis (hepatitis B and C) screening, autoimmune screening tests e.g. antinuclear antibody (ANA) and rheumatoid factor (RF) were negative. No organism was detected in blood and sputum cultures. Peripheral blood film showed marked leucocytosis with predominant eosinophils. His anti-neutrophilic cytoplasmic antibody (ANCA) was negative. Chest $x$-ray showed fibrosis in the upper zones, and it was later confirmed by high resolution CT scan (HRCT). MRI of the spine showed some degenerative changes and mild disc disease at the L3L4 region. He was started on high-dose prednisolone $(1 \mathrm{mg} / \mathrm{kg})$ and discharged well.

He was reviewed in the clinic one month later. His neurological symptoms improved following treatment. Blood investigations showed normalization of eosinophils and inflammatory markers. In addition, nerve conduction study (NCS) did not show any gross denervation. Oral Azathioprine $2.5 \mathrm{mg} / \mathrm{kg} /$ day was then added as a steroid sparing agent, coinciding with prednisolone tapering. About 7 months after the initial presentation, he presented again with worsening left foot drop. In view of recurrent symptoms, highdose steroids and intravenous cyclophosphamide were started. The latter medication was given every fortnightly for six cycles. After the completion of cyclophosphamide, most of the neurological deficits resolved except residual loss of sensation over the dorsum of both feet. His condition remained stable 18 months after the completion of cyclophosphamide.

\section{DISCUSSION}

EGPA is a rare but potentially life-threatening autoimmune disease affecting the medium to small vessels. The prevalence of EGPA ranges from $10-13 / 1,000,000$ population, with an annual incidence of about $0.5-6.8 / 1,000,000$ population. ${ }^{1}$ Most patients are between the ages of 40-60 years, with the mean age at diagnosis of about 48 years. ${ }^{1}$

According to the American College of Rheumatology (ACR) 1990 classification criteria, a person has EGPA if four of six following criteria are present, i.e. asthma, eosinophilia ( $>10 \%$ in peripheral blood), mono- or polyneuropathy, pulmonary infiltrates (non-fixed), paranasal sinus abnormality, and extravascular eosinophil infiltration on biopsy. ${ }^{2}$ The presence of four or more criteria yield a sensitivity of $85 \%$ and a specificity of $99.7 \%$. These criteria are perhaps of more use in recruiting patients for a study or trial because many cases of EGPA are diagnosed with less than four of the ACR criteria at presentation. Diagnosis of EGPA can also be supported by another laboratory evidence such as ANCA (Anti-Neutrophil Cytoplasmic Antibody). The presence of P-ANCA with antimyeloperoxidase (anti-MPO) specificity strongly supports the diagnosis of EGPA, but they are only present in about $40 \%$ of patients. ${ }^{3}$ Tissue biopsy is helpful but not essential to make a diagnosis of EGPA because findings can be non-specific and very few specimens have all the characteristic features of the disease ${ }^{1}$.
Our patient had features of EGPA i.e. presence of asthma, peripheral eosinophilia, and polyneuropathy. His ANCA was negative, as is the case in the majority of patients with EGPA.

There is no consensus regarding how EGPA should be managed. The patient's prognostic profile primarily determines the choice of the initial therapy. Guillevin et.al have introduced the Five Factor Score (FFS) to stratify patients according to their prognosis factors. ${ }^{4}$ The FFS included proteinuria $>1 \mathrm{~g} /$ day, peak serum creatinine $>140 \mu \mathrm{mol} / /(1.58 \mathrm{mg} / \mathrm{dl})$, cardiomyopathy, gastrointestinal involvement, and central nervous system involvement. The presence of each factor is given one point. The FFS score ranges from 0 to 2: a score of 0 is given when none of the factors are present, a score of 1 for one factor, and a score of 2 for two or more factors. This scoring system has also been correlated with prognosis. Patients who have one or more parameters are regarded as having poor prognosis. Patients' 5 -year overall survival was $88 \%$, $74 \%$, and $54 \%$ when 0,1, or $\geq 2$ of these parameters were present, respectively4. The FFS was revised recently in that central nervous system involvement was no longer included as a poor prognostic factor. ${ }^{5}$ Instead, the new parameters included age over 65 years, cardiac symptoms, gastrointestinal involvement, renal insufficiency (serum creatinine $>150 \mu \mathrm{mol} / \mathrm{L}$ ) and absence of ear, nose and throat manifestations.

Patients without poor-prognosis factor $(\mathrm{FFS}=0)$ usually requires corticosteroids alone as induction and maintenance therapy. A study by Ribi et.al showed that $93 \%$ of patients in this category achieved remission with corticosteroids alone. However, 35\% of them subsequently relapsed during corticosteroids tapering and therefore, required either azathioprine or cyclophosphamide to achieve disease control. ${ }^{6}$

Treatment of patients with at least one poor-prognosis factor (FFS $\geq 1$ ), on the other hand, requires a combination of corticosteroids and immunosuppressive therapy, e.g. cyclophosphamide or methotrexate. Methotrexate therapy was used in a non-randomized trial as induction but only $73 \%$ of the patients involved achieved remission.7 In addition, the study showed that its ability in preventing relapses was limited. A study by Cohen et.al showed that a combination of cyclophosphamide and corticosteroids achieved complete remission in $87.5 \%$ of 48 patients. ${ }^{8}$ Intravenous Cyclophosphamide $0.6 \mathrm{~g} / \mathrm{m} 2$ was given for 6 or 12 cycles in the study, and there were fewer relapses in the latter regimen. It is recommended that a maintenance immunosuppressive therapy using, for example, azathioprine is instituted following cyclophosphamide.

During the first presentation, our patient's FFS was 0. Therefore, he was started on high-dose corticosteroids. It was later followed by a combination of low dose corticosteroids and azathioprine. As a result of disease relapse 7 months later, he was commenced on intravenous cyclophosphamide to achieve control 
of the disease. There is an argument that supports the use of cyclophosphamide in patients without poor-prognosis factors, especially if the affected organ carries a high morbidity. ${ }^{9}$ Mononeuritis multiplex, for example, carries a significant shortand long-term morbidity if not treated aggressively. Perhaps cyclophosphamide should have been started during the initial presentation. Gayraud et al showed in their study that addition of cyclophosphamide to corticosteroids increased the efficacy of treatment in good-prognosis patients. However, the benefit was outweighed by unacceptable toxicity in this group of patients. ${ }^{10}$ There is still no consensus regarding the treatment of low risk EGPA. Perhaps the most reasonable strategy in this situation is to individualize therapy. Patient's profile, clinical presentation, and patient consent should be taken into consideration when deciding whether to use cyclophosphamide. Also, they need to be counselled regarding their clinical situation and options of treatment and side effects.

\section{CONCLUSION}

EGPA is a rare vasculitis syndrome that can present with various organ manifestations. Treatment strategy depends on whether the patient presents with or without poor-prognosis factors according to the FFS criteria. Cyclophosphamide is usually reserved for cases with poor-prognosis factors or relapse. The patient in our discussion belonged to the good-prognosis group but later required cyclophosphamide because of relapse. Mononeuritis multiplex, although is not a poor-prognosis factor, may cause significant morbidity if not treated adequately. Therefore, some reports support the use of cyclophosphamide in EGPA patients presenting with first onset mononeuritis multiplex.

\section{REFERENCES}

1. Dunogue B, Pagnoux C, Guillevin L. Churg-Strauss syndrome: Clinical symptoms, complementary investigations, prognosis and outcome, and treatment. Semin Respir Crit Care Med 2011; 32:298-309.

2. Masi AT, Hunder GG, Lie JT, et al. The American College of Rheumatology 1990 criteria for the classification of Churg-Strauss syndrome (allergic granulomatosis and angiitis). Arthritis Rheum 1990; 33:1094-100.

3. Sinico RA, Di Toma L, Maggiore U, et al. Prevalence and clinical significance of antineutrophilic cytoplasmic antibodies in Churg-Strauss syndrome. Arthritis Rheum 2005; 52:2926-35.

4. Guillevin L, Lhote F, Gayraud M, et al. Prognostic factors in polyarteritis nodosa and Churg-strauss syndrome: A prospective study in 342 patients. Medicine (Baltimore) 1996; 75:17-28.

5. Guillevin L, Pagnoux C, Seror R, et al. The Five-Factor Score revisited: assessment of prognoses of systemic necrotizing vasculitides based on the French Vasculitis Study Group (FVSG) cohort. Medicine (Baltimore) 2011; 90:19-27.

6. Ribi C, Cohen, Pagnoux C, et al. Treatment of Churg-Strauss syndrome without poor-prognosis factors: a multicenter, prospective, randomized, open-label study of seventy-two patients. Arthritis Rheum 2008; 58:586-94.

7. Metzler C, Hellmich B, Gause A, et al. Churg-Strauss syndrome - a successful induction of remission with methotrexate and unexpected high cardiac and pulmonary relapse ratio during maintenance treatment. Clin Exp Rheumatol 2004; 22:S52-S61.

8. Cohen P, Pagnoux C, Mahr A, et al. Churg-Strauss syndrome with poor-prognosis factors: a prospective multicenter trial comparing glucocorticoids and six or twelve cyclophosphamide pulses in forty-eight patients. Arthritis Rheum 2007; 57:686-93.

9. Loo KL, Ramachandran R, Chow SK, et al. Mononeuritis in Churg Strauss syndrome in Asians responding to intravenous cyclophosphamide. Rheumatology 2004; 43:252-3.

10.Gayraud M, Guillevin L, Cohen P, et al. Treatment of good-prognosis polyarteritis nodosa and Churg-Strauss syndrome: Comparisons of steroid and oral or pulse cyclophosphamide in 25 patients. Brit J Rheumatol 1997; 36:1290-7. 
HE INTERNATIONAL MEDICAL JOURNAL MALAYSIA

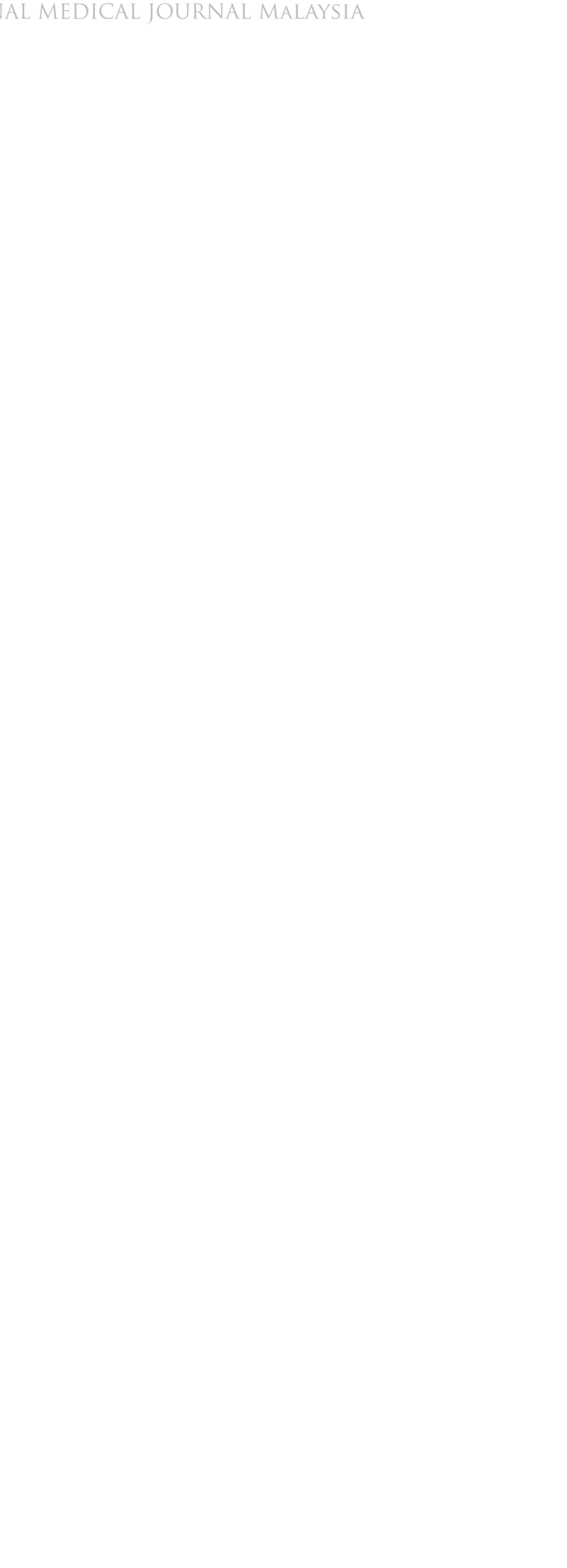

